\title{
An investigation of patients 'considered' allergic to dental local anaesthetic injection
}

\author{
Adverse reaction to dental local anaesthetic injection — ' allergy' is not the cause J. P. Rood Br Dent J2000; \\ 189: 380-384
}

\begin{abstract}
Objective
To investigate patients considered to have experienced allergic reactions to local anaesthetics administered for dental treatment.
\end{abstract}

\section{Setting}

Dental facility within a general hospital.

\section{Procedure}

Skin and intra-oral challenge tests.

\section{Results}

No patients were found to be allergic to lignocaine or prilocaine. The most likely causes of the adverse reactions were found to be psychogenic.

\section{Conclusion}

Although allergy to lignocaine (and the other amide anaesthetic agents used in dentistry) is known to be extremely rare, it continues to be suggested as a cause when adverse reactions to dental injections occur. Psychogenic reactions are the main causes of untoward events, and some can be alarming. A smaller proportion of adverse responses can be attributed to (avoidable) intravascular injections. Adverse reactions can be reduced if injections are administered carefully.

\section{Comment}

Local anaesthesia is routinely used in everyday dental practice and is the main stay of pain control during dental treatment. Allergy to local anaesthetic is considered to be rare. Ninety-seven patients were tested with skin prick tests and, of these, 75 were thought to have experienced psychogenic reactions and 22 were considered to have suffered intra-vascular injections. However, this test alone did not convince the patients or practitioners that local anaesthesia was safe for use in dentistry.

The author then embarked on not only performing intra-dermal direct challenge testing but also a 'dental' challenge test, on 44 adult patients. The protocol adopted for allergy testing was clearly outlined. This paper demonstrates the importance of a thorough history, not only from the patient, but also from the referral source in cases where an adverse event or possible allergy to local anaesthetic was suspected. Testing was performed with full resuscita- tion equipment, and a trained nurse monitored the patient throughout the observation periods. The site of the intra-dermal direct challenge test was observed for up to 1 hour and in the absence of an immediate response, a maxillary buccal infiltration of $1 \mathrm{ml}$ of $2 \%$ lignocaine with adrenaline was administered adjacent to the second pre-molar. The patient was monitored for a further 1 hour and was asked to return to the hospital if any swelling, itchiness or rash appeared after discharge. A letter was sent to the referral source with recommendations for the patient's future management. A subsequent enquiry from the dental practitioners established that 14 patients had successfully been treated with local anaesthesia.

A detailed description is given of the adverse events for all 44 patients and apart from one patient who had allergy to 'bisulphites', the remaining patients did not have an adverse response upon direct challenge with Xylocaine. The author has to be commended for the descriptions of adverse events as most of us recognise these signs in patients we have treated. Clear guidelines are given on how most adverse reactions to local anaesthetic injections can be avoided. The most frequent cause of significant reactions were psychogenic and a small number of reactions were due to intra-vascular injections. The message of this paper is clear: the true nature of the adverse event should be considered carefully and with attention to technique, such reactions can be avoided. An allergic reaction to local anaesthetic should not be suggested to the patient.

\section{A Joshi}

Clinical Lecturer/Honorary Senior Registrar, Department of Oral \& Maxillofacial Surgery, University of Manchester 